\title{
BASIC CONTOUR TRANSFORMED DENSITIES
}

\author{
A .N. Basugade \\ Professor and Head, Department of Statistics \\ Gopal Krishna Gokhale College, Kolhapur, (MS) INDIA 4160012 \\ arunb1961@gmail.com
}

Communicated : 11.03 .19

Revision : 10.04 .19

Accepted : 25.04.19

Published: 30.05 .19

\begin{abstract}
:
A New class of distributions can be generated by using a distribution or class of distributions. Some well-known methods to generate a class of distributions are transformation of variables, use of conditional distributions, introducing of additional parameters or the use of Copulas. Here we introduce Contour-Transformation; a technique based on geometric approach and use it to generate new classes of distributions. In this artical we generate densities based on the size of contours of a density function.
\end{abstract}

Key words: -Basic Contour Transformation, Unimodal, Symmetric.

\section{INTRODUCTION:}

Most of the times a proposed well-known statistical model may not give a very satisfactory fit for a collected data set. In such situations one has to make certain modifications in the model and suitable model has to be proposed. A New class of distributions can be generated by using a distribution or class of distributions. Some wellknown methods to generate a class of distributions are transformation of variables, use of conditional distributions, introducing of additional parameters or the use of Copulas.

In the literature some methods to modify a model to give a better fit for the data set have been reported. In recent days there is a trend in the literature on parametric families of asymmetric distributions making a deviation from symmetry as well as the normality assumptions. Many researchers have developed different methods to construct skewed distributions. Azzalini (1985) introduced skew Gaussian distribution in terms of a product of density function and distribution function of normal distribution. Also if $\mathrm{X}$ and $\mathrm{Y}$ are symmetric and independent random variables with probability density functions $f_{x}$ and $f_{y}$ and distribution functions $F_{X}$ and $F_{Y}$, respectively, then for any $\lambda$, $\mathrm{g}(\mathrm{y})=2 \mathrm{f}_{\mathrm{Y}}(\mathrm{y}) \mathrm{f}_{\mathrm{X}}\left(\lambda_{\mathrm{y}}\right)$ is a proper density function. This leads to a skew-normal distribution by considering $\mathrm{X}$ and $\mathrm{Y}$ to be independent and identically distributed standard normal variables. The epsilonskew normal density proposed by Mudholkar and Hutson (2000) gives better fit for data sets of heights of 219 volcanoes. Arnold, et al. (2002) have obtained a general family of multivariate densities with given contours and these include circular and elliptical densities not necessarily having the same center, but have not used transformation of the contours to generate densities. Rattihalli and Basugade (2008) have generated a class of multivariate densities by using contour transformation.

New families of distributions have also been introduced using the concepts of conditioning or truncation. When $\mathrm{X}$ has unbounded support, by conditioning on a bounded set, the natural parameter space can be enlarged yielding a new family of distributions. For further details one may refer to Rattihalli (2004).

In this article we develop models by considering transformation of contours of a probability density function (p.d.f.). Particularly we propose the densities based on Basic contour transformation.

\section{Notations and Concepts:}

Contour Transformation:Let f be a density function with modal value 0 . For $0 \leq \mathrm{u} \leq \mathrm{f}(0)$ we consider a transformation of contour $\mathrm{C}_{\mathrm{f}}(\mathrm{u})$ to $\mathrm{C}^{*}(\mathrm{u})$ such that

i) the class $\left\{\mathrm{C}^{*}(\mathrm{u}): 0 \leq \mathrm{u} \leq \mathrm{f}(0)\right\}$ is non-increasing in $\mathrm{u}$ and

ii) $\wedge\left(\mathrm{C}_{\mathrm{f}}(\mathrm{u})\right)=\wedge\left(\mathrm{C}^{*}(\mathrm{u})\right), 0 \leq \mathrm{u} \leq \mathrm{f}(0)$.

where, $\wedge$ is the Lebesque measure on $\mathfrak{R}^{\mathrm{k}}$. Note that corresponding to such a class $\mathbf{C}^{*}=\left\{\mathrm{C}^{*}(\mathrm{u}): 0 \leq \mathrm{u} \leq \mathrm{f}(0)\right\}$ there exists a function $\mathrm{f}^{*}$ (say) given by $\mathrm{f}^{*}(\mathrm{x})=$ sup $\left\{\mathrm{u}: \mathrm{x} \in \mathrm{C}^{*}(\mathrm{u})\right\}$ such that $\mathrm{C}^{*}(\mathrm{u})=\mathrm{C}_{\mathrm{f}^{*}}(\mathrm{u}), 0 \leq \mathrm{u} \leq \mathrm{f}(0)$. It

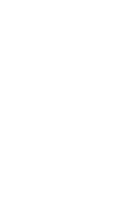


is to be noted that, from condition (1), corresponding to the class $\mathbf{C}^{*}$, there exists a function $\mathrm{f}^{\star}$. Since

$\int_{R^{k}} f^{*}(x) d x=\int_{R^{k}}\left[\int_{0}^{f^{*}(x)} d u\right] d x=\int_{0}^{f^{*}(x)}\left[\int_{0<u \leq f^{*}(u)} d u\right] d x=\int_{0}^{f^{*}\left(M^{*}\right)}\left[\int_{x, f^{*}(x) \geq u} d x\right] d u=\int_{0}^{f^{*}\left(M^{*}\right)} \Lambda\left(c^{*}(u)\right) d u$ $=\int_{0}^{f(0)} \Lambda(c(u)) d u=\int_{0}^{f(0)}\left[\int_{x: f(x) \geq u} d x\right] d u=\int_{R^{k}}\left[\int_{0}^{f(x)} d u\right] d x=\int_{R^{k}} f(x) d x=1$.

the function $\mathrm{f}^{*}$ is p.d.f.

The requirement (1) is a major constraint on a CT. An arbitrary

transformed class of contours may not correspond to a function.

For $\mathrm{u} \in \mathfrak{R}$ define

$h(u)= \begin{cases}\wedge\left(\mathrm{C}_{\mathrm{f}}(\mathrm{u})\right), & 0 \leq \mathrm{u} \leq \mathrm{f}(0) \\ 0, & \text { otherwise. }\end{cases}$

For an arbitrary (not necessarily continuous) monotone decreasing function $\mathrm{t}(\mathrm{x})$, we define its inverse as $\mathrm{t}^{-1}(\mathrm{u})=\sup \{\mathrm{x}: \mathrm{t}(\mathrm{x}) \geq \mathrm{u}\}$.

The function

$\mathrm{g}(\mathrm{x})=\mathrm{h}^{-1}(\mathrm{x})$

is also a p.d.f.,

\section{Basic Contour Transformation:}

The CT that transforms $\mathrm{C}_{\mathrm{f}}(\mathrm{u})$ to $\left(0, \wedge\left(\mathrm{C}_{\mathrm{f}}(\mathrm{u})\right)\right)$, for $\mathrm{u} \geq$ 0 is called the Basic Contour Transform. The p.d.f. corresponding to the contours $\left(0, \wedge\left(\mathrm{C}_{\mathrm{f}}(\mathrm{u})\right)\right)$ be called as the Basic Contour Transformed density of $\mathrm{f}$. The function $g$ (.) defined in (4) is Basic Contour Transformed density of f. Following figure shows the graphs of the function $h$ and $g$, the basic transform of $f$.

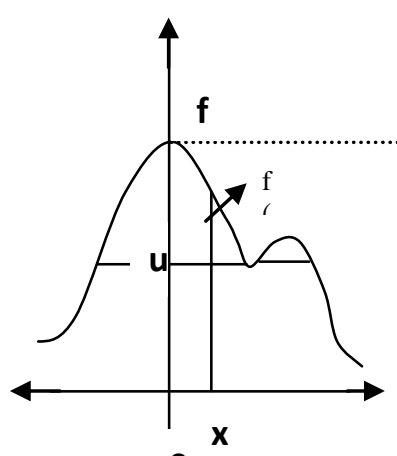

0

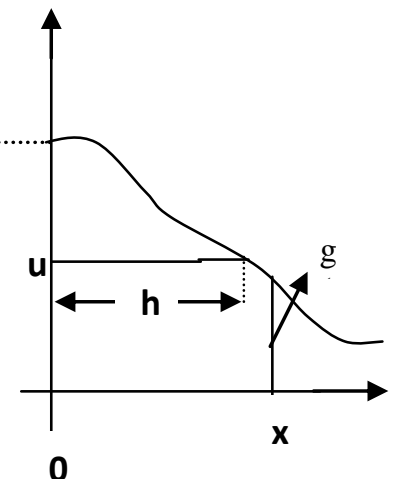

Fig. 1: Graphs of the furıcion $\mathrm{h}$ and $\mathrm{g}$, the basic transform of $\mathrm{f}$.

For example, let $f$ correspond to $p$. d. $f$. of univariate normal random variable with mean $\theta$ and variance $\sigma^{2}$. Here

$\mathrm{C}_{\mathrm{f}}(\mathrm{u})=(\theta-\sigma \sqrt{-2 \log (\sigma \mathrm{u} \sqrt{2 \pi})}, \theta+\sigma \sqrt{-2 \log (\sigma \mathrm{u} \sqrt{2 \pi})})$ $\wedge\left(\mathrm{C}_{\mathrm{f}}(\mathrm{u})\right)=2 \sigma \sqrt{-2 \log (\sigma \mathrm{u} \sqrt{2 \pi)}}$,
Hence

$$
h(u)=2 \sigma \sqrt{-2 \log (\sigma u \sqrt{2 \pi})}, \quad \text { for } 0 \leq u \leq f(0) .
$$

$g(x)=\left\{\begin{array}{cc}\left(1 / \sigma \sqrt{2 \pi)} \exp \left\{-(1 / 2)[(x / 2) / \sigma]^{2}\right\},\right. & x>0 \\ 0, & \text { otherwise }\end{array}\right.$ In general if $\mathrm{f}$ is symmetric p. $\mathrm{d}$. $\mathrm{f}$. about $\theta$ then $g(x)=\left\{\begin{array}{cl}f(\theta+x / 2), & x>0 \\ 0, & \text { otherwise. }\end{array}\right.$

We note that if $\mathrm{f}$ is the p.d.f. of $\mathrm{X}$, then $\mathrm{g}$ is neither the p.d.f. of $\mathrm{I} x-\theta \mathrm{I}$ nor that of

But it is the p.d.f. of $\max \{2(|x|-\theta), 0\}$.

\section{Basic contour transformed density when $f$ is symmetric about 0 :}

Let $\mathrm{f}$ is a unimodal symmetric density function with modal value 0 .

Define $\mathrm{k}(\mathrm{x})$ as

$$
\mathrm{k}(\mathrm{x})=\mathrm{f}(|\mathrm{x}|), \quad \mathrm{x}>0 .
$$

Here
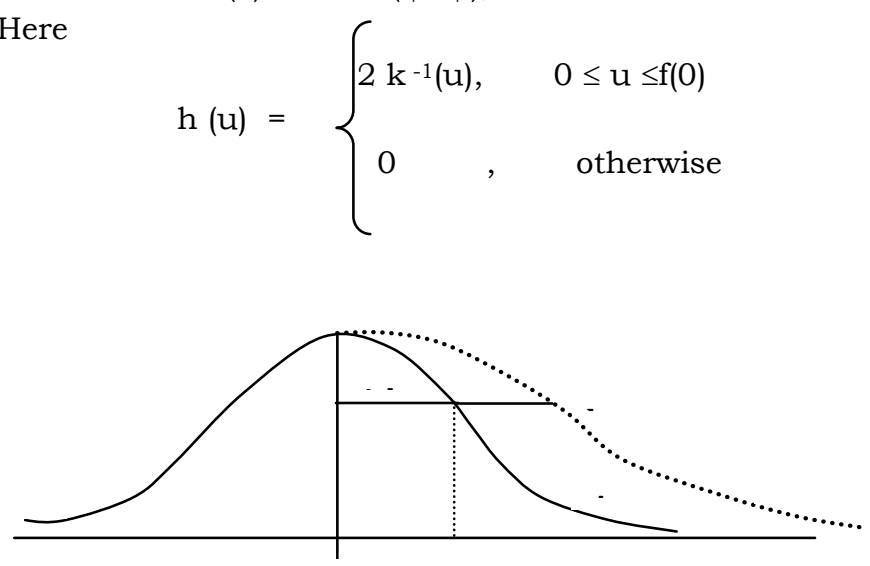

Fig. 2: Graph of $\mathrm{k}(\mathrm{x})$ and $\mathrm{h}(\mathrm{u})$

Thus the Basic contour transformed density $\mathrm{f}^{*}$ is

$$
f^{*}(x)= \begin{cases}(x / 2), & x>0 \\ 0, & \text { otherwise }\end{cases}
$$

\section{Illustrations:}

\section{a) Standard Normal Distribution:}

Let $f(x)=\left(1 / \sqrt{2 \pi)} \exp \left\{-x^{2} / 2\right\}, \quad-\infty \leq x \leq \infty\right.$

$\mathrm{u}=\mathrm{f}(\mathrm{x})$ gives $\mathrm{u}=\left(1 / \sqrt{2 \pi)} \exp \left\{-\mathrm{x}^{2} / 2\right\}\right.$ and

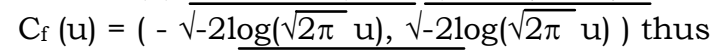

$h(u)=\left\{\begin{array}{cl}-2 \sqrt{-2 \log (\sqrt{2 \pi u}),} & 0 \leq u \leq(1 / \sqrt{2} \overline{\pi)} \\ 0, & \text { otherwise }\end{array}\right.$

and $\mathrm{x}=\mathrm{h}(\mathrm{u})$ gives $\mathrm{u}=(1 / \sqrt{ } 2 \pi) \exp \left\{\mathrm{x}^{2} / 8\right\}, \mathrm{x}>0$ 
Thus the Basic contour transformed density $\mathrm{f}^{*}(\mathrm{x})$ is given by

$f^{*}(x)= \begin{cases}\left(1 / \sqrt{2 \pi)} \exp \left\{-x^{2} / 8\right\},\right. & x>0 \\ 0, & \text { otherwise }\end{cases}$

\section{b) Student's t distribution:}

$f(x)=\frac{1}{n^{1 / 2} \beta(n / 2,1 / 2)\left(1+x^{2} / n\right)^{(n+1) / 2}}, \quad-\infty<x<\infty$

$\mathrm{u}=\mathrm{f}(\mathrm{x})$

gives

$x= \pm(\sqrt{n})^{n /(n+1)} \frac{\left[1-(\beta u \sqrt{n})^{2 /(n+1)}\right]^{1 / 2}}{(\beta u)^{1 /(n+1)}}$

and

$C_{f}(u)=\left(-(\sqrt{n})^{n /(n+1)} \frac{\left[1-(\beta u \sqrt{n})^{2 /(n+1)}\right]^{1 / 2}}{(\beta u)^{1 /(n+1)}},(\sqrt{n})^{n /(n+1)} \frac{\left[1-(\beta u \sqrt{n})^{2 /(n+1)}\right]^{1 / 2}}{(\beta u)^{1 /(n+1)}}\right)$

thus

$h(u)= \begin{cases}2(\sqrt{n})^{n /(n+1)} \frac{\left[1-(\beta u \sqrt{n})^{2 /(n+1)}\right]^{1 / 2}}{(\beta u)^{1 /(n+1)}}, & 0<x<\frac{1}{n^{1 / 2} \beta(n / 2,1 / 2),} \\ 0, & \text { otherwise }\end{cases}$

and $\mathrm{x}=\mathrm{h}(\mathrm{u})$ gives $\mathrm{u}=$

$\frac{1}{n^{1 / 2} \beta(n / 2,1 / 2)\left(1+x^{2} / 4 n\right)^{(n+1) / 2}}, \mathrm{x}>0$.

Thus the Basic contour transformed density $\mathrm{f}^{*}(\mathrm{x})$ is given by

$\mathrm{f}^{*}(\mathrm{x})=\left\{\begin{array}{cc}\frac{1}{n^{1 / 2} \beta(n / 2,1 / 2)\left(1+x^{2} / 4 n\right)^{(n+1) / 2}}, & \mathrm{x}>0 \\ 0, & \text { otherwise }\end{array}\right.$

5. Basic contour transformed densities when $f$ is symmetric about $\theta$ :

\section{c) Laplace distribution:}

Let $\mathrm{f}(\mathrm{x})=(1 / 2 \theta) \exp \{-|\mathrm{x} / \theta|\}, \quad-\infty \leq \mathrm{x} \leq \infty$ then $\mathrm{u}=\mathrm{f}(\mathrm{x})$ gives $\mathrm{u}=(1 / 2 \theta) \exp \{-|\mathrm{x} / \theta|\}$ and

$\mathrm{C}_{\mathrm{f}}(\mathrm{u})=(\theta \log (2 \theta \mathrm{u}),-\theta \log (2 \theta \mathrm{u}))$ thus $h(u)=\left\{\begin{array}{cc}-2 \theta \log (2 \theta u), & 0 \leq u \leq(1 / 2 \theta) \\ 0 \quad, & \text { otherwise }\end{array}\right.$

$\mathrm{x}=\mathrm{h}(\mathrm{u})$ gives $\mathrm{u}=(1 / 2 \theta) \exp \{-(\mathrm{x} / 2 \theta)\}, \mathrm{x}>0$.

Thus the Basic contour transformed density $\mathrm{f}^{*}(\mathrm{x})$ is given by

$\mathrm{f}^{\prime}(\mathrm{x})=\left\{\begin{array}{cc}(1 / 2 \theta) \exp \{-(\mathrm{x} / 2 \theta)\}, & \mathrm{x}>0 \\ 0, & \text { otherwise }\end{array}\right.$

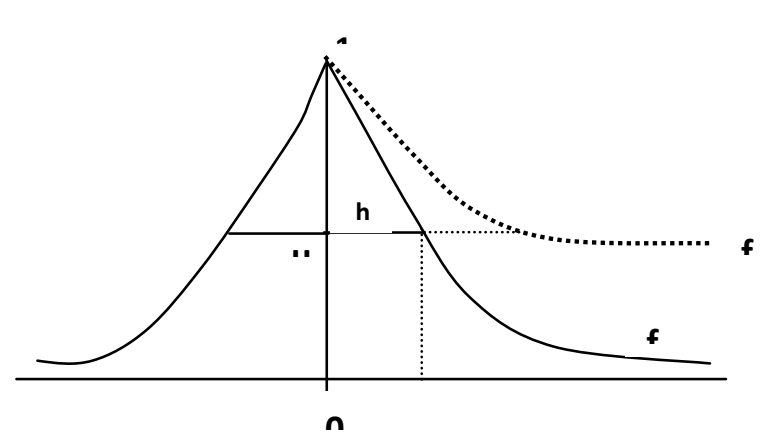

Fig. 3: Graphs of $\mathrm{f}$ and $\mathrm{f}^{*}$

The first two moments are $\mathrm{E}(\mathbf{x})=\mathbf{2} \theta$,

$\mathrm{E}\left(\mathrm{x}^{2}\right)=8 \theta^{2}$ and Variance is $\mathrm{V}(\mathrm{x})=4 \theta^{2}$

c) Cauchy distribution:

$\mathrm{f}(\mathrm{x})=\frac{1}{\pi}\left[\frac{\theta}{\theta^{2}+x^{2}}\right], \quad-\infty<x<\infty$

$\mathrm{u}=\mathrm{f}(\mathrm{x})$ gives $\mathrm{u}=\frac{1}{\pi}\left[\frac{\theta}{\theta^{2}+x^{2}}\right]$ thus $\mathrm{x}= \pm \sqrt{\frac{\theta}{\pi u}-\theta^{2}}$ and

$\mathrm{C}_{\mathrm{f}}(\mathrm{u})=\left(-\sqrt{\frac{\theta}{\pi u}-\theta^{2}}, \sqrt{\frac{\theta}{\pi u}-\theta^{2}}\right)$, thus

$\mathrm{h}(\mathrm{u})= \begin{cases}2 \sqrt{\frac{\theta}{\pi u}-\theta^{2}} & 0 \leq \mathrm{u} \leq(1 / \pi \theta) \\ 0, & \text { otherwise }\end{cases}$

and $\mathrm{x}=\mathrm{h}(\mathrm{u})$ gives $\mathrm{u}=\frac{1}{\pi}\left[\frac{\theta}{\theta^{2}+\frac{x^{2}}{4}}\right], \quad \mathrm{x}>0$

Thus the Basic contour transformed density $\mathrm{f}^{*}(\mathrm{x})$ is given by 
$\mathrm{f}^{*}(\mathrm{x})=\left\{\begin{array}{lr}\frac{1}{\pi}\left[\frac{\theta}{\theta^{2}+\frac{x^{2}}{4}}\right], & \mathrm{x}>0 \\ 0, & \text { otherwise }\end{array}\right.$

e) Pareto distribution:

$f(x)=\frac{\beta}{x^{\beta+1}}, \quad 1<x<\infty, \quad \beta>0$

$\mathrm{u}=\mathrm{f}(\mathrm{x})$ gives $\mathrm{u}=\beta / \mathrm{x}^{\beta+1}$ and $\mathrm{x}=[\beta / \mathrm{u}]^{1 /(\beta+1)}$

thus $\mathrm{C}_{\mathrm{f}}(\mathrm{u})=\left(1,[\beta / \mathrm{u}]^{1 /(\beta+1)}\right)$ and

$\wedge\left(\mathrm{C}_{\mathrm{f}}(\mathrm{u})\right)=[\beta / \mathrm{u}]^{1 /(\beta+1)}-1$,

$h(u)=\left\{\begin{array}{cc}{[\beta / u]^{1 /(\beta+1)-1,}} & 0 \leq \mathrm{u} \leq \beta . \\ 0, & \text { otherwise }\end{array}\right.$

$\mathrm{x}=\mathrm{h}(\mathrm{u})$ gives $\mathrm{u}=\beta /[\mathrm{x}+1]^{\beta+1}, \mathrm{x}>0$.

Thus the Basic contour transformed density $\mathrm{f}^{*}(\mathrm{x})$ is given by

$f^{*}(x)=\left\{\begin{array}{cc}\beta /[x+1]^{\beta+1}, & x>0 . \\ 0, & \text { otherwise }\end{array}\right.$

\section{CONCLUSION:}

By using Basic contour transformations we can modify the densities which are symmetric about zero and also symmetric about $\theta$. These modified densities can be used for further data analysis.

\section{REFERENCES:-}

Arnold, B., Castillo, E. and Sarabia, J.M. (2002).Multivariate Models Involving Conditional Specification.Journal of the Indian Society for Probability and Statistics, Vol.6, (97-121).

Azzalini, A.(1985 ). A Class of distributions which includes thenormal ones.Scandinavian Journal of Statistics, 12, (171-178).

Azzalini, A. and Dalla Valle A. (1996).The Multivariate Skew-normal distribution.Biometrica, 84 / 4, (715-726).
Lai C.D. (2004). Constructions of Continuous Bivariate Distributions.Jr. of Indian Society for Probability and Statistics. Vol. 8 (21-44).

Mudholkar G.S. and Hutson A. D. (2000). The epsilon- skew normal

distribution for analyzing near normal dated. Jr. of Statistical

Planning and Inference, 83,(291-309).

Nelsen, R.B. (1999). An Introduction to Copulas.Springer, New York.

Rattihalli R.N. (2004). An unpublished Report of a Research Project, Unpublished ReportSubmitted to University Grant Commission, New Delhi.

Rattihalli R.N., and Basugade A. N. (2008), "Generation of MultivariateDensities," Statistical Science and Interdisciplinary Research, Vol. 4, World Scientific Review, pp 185-196. 